\title{
Uncovering the microbiome of invasive sympatric European brown hares and European rabbits in Australia
}

\author{
Somasundhari Shanmuganandam ${ }^{1,2}$, Yiheng Hu ${ }^{1}$, Tanja Strive ${ }^{2,3}$, Benjamin Schwessinger $^{1}$, Robyn N Hall ${ }^{\text {Corresp. } 2,3}$ \\ ${ }^{1}$ Research School of Biology, Australian National University, Acton, ACT, Australia \\ 2 Health \& Biosecurity, Commonwealth Scientific and Industrial Research Organisation, Acton, ACT, Australia \\ 3 Centre for Invasive Species Solutions, Bruce, ACT, Australia \\ Corresponding Author: Robyn N Hall \\ Email address: robyn.hall@csiro.au
}

Background. European brown hares (Lepus europaeus) and European rabbits (Oryctolagus cuniculus) are invasive pest species in Australia, with rabbits having a substantially larger environmental impact than hares. As their spatial distribution in Australia partially overlaps, we conducted a comparative microbiome study to determine how the composition of gastrointestinal microbiota varies between these species, since this may indicate species differences in diet, physiology, and other internal and external factors.

Methods. We analysed the faecal microbiome of nine wild hares and twelve wild rabbits from a sympatric peri urban reserve in Canberra, Australia, using a 16S rRNA amplicon-based sequencing approach. Additionally, we compared the concordance between results from Illumina and Nanopore sequencing platforms.

Results. We identified significantly more variation in faecal microbiome composition between individual rabbits compared to hares, despite both species occupying a similar habitat. The faecal microbiome in both species was dominated by the phyla Firmicutes and Bacteroidetes, typical of many vertebrates. Many phyla, including Actinobacteria, Proteobacteria, and Patescibacteria, were shared between rabbits and hares. In contrast, bacteria from phylum Verrucomicrobia were present only in rabbits, while phyla Lentisphaerae and Synergistetes were represented only in hares. We did not identify phylum Spirochetes in Australian hares; this phylum was previously shown to be present at high relative abundance in European hare faecal samples. These differences in the composition of faecal microbiota, especially in rabbits, may be indicative of less discriminate foraging behaviour in rabbits, which in turn may enable them to adapt quicker to new environments, and may reflect the severe environmental impacts that this species has in Australia. 


\section{Uncovering the microbiome of invasive sympatric} 2 European brown hares and European rabbits in

\section{Australia}

4

5

6 Somasundhari Shanmuganandam ${ }^{1,2}$, Yiheng $\mathrm{Hu}^{1}$, Tanja Strive ${ }^{2,3}$, Benjamin Schwessinger ${ }^{1}$,

7 Robyn N. Hall ${ }^{2,3}$

8

$9 \quad{ }^{1}$ Research School of Biology, The Australian National University, Acton, ACT, Australia

$10{ }^{2}$ Health \& Biosecurity, Commonwealth Scientific and Industrial Research Organisation, Acton,

11 ACT, Australia

$12{ }^{3}$ Centre for Invasive Species Solutions, Bruce, ACT, Australia

13

14 Corresponding Author:

15 Robyn Hall

1611 Clunies Ross St, Acton, ACT, 2601, Australia

17 Email address: robyn.hall@csiro.au 
18

19

20

21

22

23

24

25

26

27

28

29

30

31

32

33

34

35

36

37

38

39

40

41

42

43

44

45

46

47

48

49

50

51

52

53

54

55

56

57

\section{Abstract}

Background. European brown hares (Lepus europaeus) and European rabbits (Oryctolagus cuniculus) are invasive pest species in Australia, with rabbits having a substantially larger environmental impact than hares. As their spatial distribution in Australia partially overlaps, we conducted a comparative microbiome study to determine how the composition of gastrointestinal microbiota varies between these species, since this may indicate species differences in diet, physiology, and other internal and external factors.

Methods. We analysed the faecal microbiome of nine wild hares and twelve wild rabbits from a sympatric peri urban reserve in Canberra, Australia, using a 16S rRNA amplicon-based sequencing approach. Additionally, we compared the concordance between results from Illumina and Nanopore sequencing platforms.

Results. We identified significantly more variation in faecal microbiome composition between individual rabbits compared to hares, despite both species occupying a similar habitat. The faecal microbiome in both species was dominated by the phyla Firmicutes and Bacteroidetes, typical of many vertebrates. Many phyla, including Actinobacteria, Proteobacteria, and Patescibacteria, were shared between rabbits and hares. In contrast, bacteria from phylum Verrucomicrobia were present only in rabbits, while phyla Lentisphaerae and Synergistetes were represented only in hares. We did not identify phylum Spirochetes in Australian hares; this phylum was previously shown to be present at high relative abundance in European hare faecal samples. These differences in the composition of faecal microbiota, especially in rabbits, may be indicative of less discriminate foraging behaviour in rabbits, which in turn may enable them to adapt quicker to new environments, and may reflect the severe environmental impacts that this species has in Australia.

\section{Introduction}

In a new environment, non-native species must face several barriers to first invade and then become established. They must quickly adapt to new environmental conditions while also competing with native species for food, shelter, and water $(1,2)$. European rabbits (Oryctolagus cuniculus) and European brown hares (Lepus europaeus) are lagomorphs in the family Leporidae and are both non-native species in Australia, originally introduced from Europe in 1859 (3). European rabbits rapidly colonised most of the Australian continent and are considered to be one of Australia's worst invasive vertebrate pests due to land degradation and competition with native animals and livestock (4-6). Despite foraging over wider areas, the impacts of European brown hares are less severe, although they are still considered to be a pest species $(6,7)$. The specific factors underlying differences in colonising potential and impacts of each species are likely multifactorial, including differences in host physiology, reproductive strategies, behaviour, diet, and interaction with commensal and pathogenic microbes. 
58 Recent advancements in sequencing technologies have highlighted the importance of interactions

59

60

61

62

63

64

65

66

67

68

69

70

71

72

73

74

75

76

77

78

79

80

81

82

83

84

85

86

87

88

89

90

91

92

93

94

95

96

97

between the gut microbiome, host behaviour and physiology, and dietary preferences (8-13). Although the gastrointestinal microbiome of both rabbits and hares have previously been investigated, most studies to date were conducted on domestic rabbit populations in either Europe or China (14-24). Evaluating the microbial diversity in the gastrointestinal tract of wild hares and rabbits in Australia may provide additional insights as to how these two species differ, especially in terms of diet and/or metabolism (25). Furthermore, identifying differences between the gastrointestinal microbiota of lagomorphs in their native range compared to their introduced range may lead to novel insights into how to more sustainably manage introduced populations, for example, by identifying pathogens that are absent in the introduced range that may be suitable for evaluation as biocontrol agents (25).

The microbiome can be investigated through a range of both traditional culture-dependent and less biased culture-independent techniques, including high throughput sequencing (26-31). Among various sequencing strategies, taxonomic profiling based on the $16 \mathrm{~S}$ ribosomal RNA (rRNA) gene is widely used for estimating the bacterial and archaeal diversity in tissue or environmental samples due to the availability of well-curated databases such as SILVA (32). Broadly, high-throughput sequencing can be classified as either short-read sequencing, using the Illumina platform, or long-read sequencing, using platforms such as PacBio or Oxford Nanopore $(33,34)$. Although all these platforms have previously been used to conduct 16S rRNA microbial profiling studies, direct comparisons of these methods are limited (35).

To investigate the differences between the gastrointestinal microbiome of Australian wild rabbits and hares we conducted 16S rRNA sequencing on faecal samples collected from sympatric populations of these species using both Illumina and Oxford Nanopore sequencing platforms. The gastrointestinal microbial diversity of Australian lagomorphs was then compared to that observed in European populations to explore differences between populations in their native and invasive ranges.

\section{Materials \& Methods}

\section{Sample collection}

Faecal samples were collected at necropsy from nine wild European brown hares (Lepus europaeus) and twelve wild European rabbits (Oryctolagus cuniculus) of both sexes (Table S1). Although caecal contents may more accurately reflect the microbiota involved in digestion in these species, these samples were not collected for this study. The hares and rabbits used in this study were mostly adults or young adults, they were outwardly healthy, and were shot as part of routine vertebrate pest control operations in Mulligans Flat, ACT (-35.164, 149.165), between January and September 2016. Sample collection was approved by Barry Richardson, Oliver Orgill, and the ACT Parks and Conservation Service rangers of Mulligan's Flat Woodland

Peer) reviewing PDF | (2020:04:47575:1:1:NEW 5 Jun 2020) 
98

99

100

101

102

103

104

105

106

107

108

109

110

111

112

113

114

115

116

117

118

119

120

121

122

123

124

125

126

127

128

129

130

131

132

133

134

135

136

137

Sanctuary. Mulligans Flat Nature Reserve comprises 781 hectares of box-gum grassy woodland with a sparse to moderately dense ground cover of grasses, herbs, and shrubs. The reserve is fenced, preventing immigration and emigration of most animals. Rabbits and hares were shot from a vehicle using a 0.22 -caliber rifle targeting the head or chest. Faecal pellets from the descending colon were collected during post-mortem and stored at $-20^{\circ} \mathrm{C}$. All sampling was conducted according to the Australian Code for the Care and Use of Animals for Scientific Purposes as approved by CSIRO Wildlife and Large Animal Ethics Committee (approvals \#1215 and \#16-02).

\section{Genomic DNA extraction}

Genomic DNA was extracted from $50 \mathrm{mg}$ of faecal pellets using the DNeasy Blood \& Tissue kit as per manufacturer's instructions and described in detail at https://www.protocols.io/view/genomic-dna-extraction-from-animal-faecal-tissues-6bbhain (Qiagen, Chadstone Centre, Victoria). Hare and rabbit samples were processed separately, and reagent-only controls (ROC) were included with each set of extractions. All samples were quantified and evaluated for integrity using the Qubit dsDNA Broad Range assay kit and the Qubit Fluorometer v2.0 (Thermo Fisher Scientific, Waltham, MA) and Nanodrop ND-1000 (Thermo Fisher Scientific). Genomic DNA samples were stored at $-20{ }^{\circ} \mathrm{C}$.

\section{Illumina library preparation, sequencing, and bioinformatics analysis}

We amplified the V3-V4 region of the 16S rRNA gene ( $\sim 460 \mathrm{bp})$ from genomic DNA and ROC according to the Illumina protocol for 16S Metagenomic Sequencing Library Preparation using a dual-indexing strategy, with modifications (36). Briefly, initial PCR reactions $(25 \mu \mathrm{l})$ were performed for each sample (including ROC) using 2x Platinum SuperFi PCR Master Mix (Thermo Fisher Scientific), $0.5 \mu \mathrm{M}$ of forward (5'-

\section{TCGTCGGCAGCGTCAGATGTGTATAAGAGACAGCCTACGGGNGGCWGCAG- 3')} and reverse primer $\left(5^{\prime}-\right.$

\section{GTCTCGTGGGCTCGGAGATGTGTATAAGAGACAGGACTACHVGGGTATCTAATC}

C -3') (overhang adapter sequence highlighted in bold) and 4.6 ng of genomic DNA as described at https://www.protocols.io/view/library-preparation-protocol-to-sequence-v3-v4-reg-6i7hchn. A 'no template control' (NTC) was included during reaction setup. Cycling conditions were: $98^{\circ} \mathrm{C}$ for $30 \mathrm{sec}$, followed by $25 \mathrm{cycles}$ of $98^{\circ} \mathrm{C}$ for $10 \mathrm{sec}, 55^{\circ} \mathrm{C}$ for $15 \mathrm{sec}$, and $72{ }^{\circ} \mathrm{C}$ for $30 \mathrm{sec}$, with a final extension of $10 \mathrm{~min}$ at $72^{\circ} \mathrm{C}$. PCR products were confirmed by agarose gel electrophoresis before being purified using AMPure XP beads (Beckman Coulter, Indianapolis, $\mathrm{IN}$ ) at a $1 \mathrm{x}$ ratio as described previously (36). Each amplicon was then dual-indexed with unique DNA barcodes using the Nextera XT index kit (N7XX and S5XX, Illumina, San Diego, CA) for PCR-based barcoding (36). For each $50 \mu \mathrm{l}$ PCR reaction, we used $5 \mu \mathrm{l}$ of each index (i7 and i5), and $5 \mu \mathrm{l}$ of the first PCR product. Cycling conditions were as described above but limited to eight cycles. Final libraries (including ROC and NTC) were purified with AMPure XP beads (Beckman Coulter), validated using the Tapestation D1000 high sensitivity assay (Agilent 
138 Technologies, Santa Clara, CA) and the Qubit High Sensitivity dsDNA assay kit (Thermo Fisher 139 Scientific), pooled at equimolar concentrations, and sequenced on an Illumina MiSeq using 600cycle v3 chemistry (300 bp paired-end) at CSIRO, Black Mountain, Canberra.

Illumina fastq reads were analysed in QIIME 2-2019.7 software (37). Raw fastq reads were quality filtered (i.e. filtered, dereplicated, denoised, merged, and assessed for chimaeras) to produce amplicon sequence variants (ASV) using the DADA2 pipeline via QIIME2 (38). The DADA2 generated feature table was filtered to remove ASVs at a frequency less than two, and remaining ASVs were aligned against the SILVA_132 (April, 2018) reference database using the same reference database using BLASTn (2.2.28) through the command line interface (CLI) using an e-value cut-off of 1e-90 (Table S2). We processed and exported BLASTn outputs into QIIME2 to perform taxonomic analysis. All scripts used were deposited at https://github.com/SomaAnand/Hare _rabbit_microbiome. Raw sequence data were deposited in the sequence read archive of NCBI under accession number PRJNA576096.

153

The microbial diversity and richness in individual samples was estimated using alpha diversity metrics (Shannon index and observed OTU), and the variation in overall composition between rabbits and hares was measured using beta diversity metrics (Bray-Curtis dissimilarity) after rarefaction at a subsampling depth of 149,523 using the q2-diversity pipeline within QIIME2 $(42,43)$. This subsampling depth was chosen based on the sample (excluding one ROC (rabbit) and PCR NTC) with the lowest number of reads.

160

\section{Nanopore library preparation, sequencing, and bioinformatics analysis}

163

164

165

166

167

168

169

170

171

172

173

174

175

176

177

The entire 16S rRNA gene ( 1500 bp) was targeted for sequencing using the Nanopore MinION sequencing platform. We amplified the $16 \mathrm{~S}$ rRNA gene from all genomic DNA samples using the universal primers $27 \mathrm{~F}$ and $1492 \mathrm{R}$ (44). PCR reactions $(100 \mu \mathrm{l})$ were performed for each sample using 2x Platinum SuperFi PCR master mix (Thermo Fisher Scientific), $0.4 \mu \mathrm{M}$ each primer, and $11.5 \mathrm{ng}$ genomic DNA as described at https:/www.protocols.io/view/librarypreparation-protocol-to-sequence-full-leng-6j6hcre. Cycling conditions were: $98^{\circ} \mathrm{C}$ for $30 \mathrm{sec}$, followed by 28 cycles of $98^{\circ} \mathrm{C}$ for $10 \mathrm{sec}, 55^{\circ} \mathrm{C}$ for $15 \mathrm{sec}$, and $72{ }^{\circ} \mathrm{C}$ for $40 \mathrm{sec}$, with a final extension of $5 \mathrm{~min}$ at $72{ }^{\circ} \mathrm{C}$. $\mathrm{PCR}$ products were confirmed by agarose gel electrophoresis before being purified with AMPure XP beads (Beckman Coulter), validated using the Tapestation D1000 high sensitivity assay (Agilent Technologies), and quantified using the Qubit High Sensitivity dsDNA assay kit (Thermo Fisher Scientific). For library preparation we used the ligation sequencing kit 1D (SQK-LSK108) in combination with the native barcoding kit 1D (EXP-NBD103) (Oxford Nanopore Technologies, Oxford, UK) as per the manufacturer's protocol, except $500 \mathrm{ng}$ of input DNA per sample was used for end preparation (45). For the addition of barcodes, $80 \mathrm{ng}$ of end-prepped DNA was used as input, and barcoded samples were pooled in equimolar concentrations to obtain at least $400 \mathrm{ng}$ of pooled DNA. We used a final 
178 library amount of $200 \mathrm{ng}$ to obtain maximum pore occupancy on a MinION R.9.4.1 flow cell.

179 Two MinION flowcells were used to run all samples and each flow cell had a mix of hare and 180 rabbit samples to control for potential batch effects.

181

182

183

184

185

186

187

188

189

190

191

192

193

194

195

196

197

198

199

200

201

202

203

204

205

206

207

208

209

210

211

212

213

214

215

216

217

Nanopore raw reads in fast5 format were demultiplexed using deepbinner (46). Basecalling, adapter trimming, and conversion into fastq format was performed in Guppy 2.3.7 (Oxford Nanopore Technologies). BLASTn was used to locally align $16 \mathrm{~S}$ sequences with a quality score higher than seven against the SILVA_132 reference database. Top hits were exported in Biological Observation Matrix (BIOM) format and imported into QIIME2 for subsequent analyses. Nanopore data was rarefied at a subsampling depth of 109,435 for diversity analysis based on the sample with the fewest Nanopore reads, in order to mimic the Illumina workflow. This rarefied dataset was then used to analyse the taxonomic results as per the Illumina workflow described above. Species level taxonomic classification was performed through the EPI2ME platform (Oxford Nanopore) (Table S2). Raw sequence data were deposited in the sequence read archive (SRA) of NCBI under accession number PRJNA576096.

\section{Statistical analysis}

We assessed whether the bacterial diversity of hares and rabbits was statistically different by using permutation-based statistical testing (PERMANOVA) via the QIIME diversity beta-groupsignificance pipeline within QIIME2 for both Illumina and Nanopore datasets (43). Statistical significance in alpha diversity was estimated using the nonparametric Kruskal-Wallis test within the R package 'ggpubr' (47). We also evaluated statistical differences between hare and rabbit faecal samples for each observed bacterial phyla using a combination of multiple tests. We performed analysis of variance (ANOVA) to estimate the variance of both populations and Student's t-test to identify statistical differences between the means of both groups. Estimated pvalues were corrected using the Benjamini-Hochberg method to control the False Discovery Rate for multiple hypothesis testing. Pearson correlation coefficients were calculated to assess the correlation between Illumina and Nanopore sequencing results using the scipy.stats package in Python.

\section{Results}

\section{Interrogating hare and rabbit faecal microbiota using 16S rRNA sequencing}

We performed Illumina short-read sequencing of the 16S rRNA V3-V4 region and Nanopore long read sequencing of the entire 16S rRNA gene on faecal samples of wild hares and rabbits living sympatrically in a periurban nature reserve in Australia. Short-read sequencing of 24 samples through the Illumina MiSeq pipeline produced an aggregate of 14,559,822 reads greater than Q30 (mean 501,908 reads per sample, excluding ROC and NTC). Reads were converted to ASVs, which were assigned using the SILVA_132 reference database via BLAST+ in QIIME2. This produced 6,662 unique features at a frequency greater than two. Long-read sequencing of 
21821 samples (no ROCs or NTC due to lack of amplification) using the Nanopore MinION 219 platform produced an aggregate of 6,544,770 reads (mean of 311,656 reads per sample) above a 220 quality threshold of seven across two MinION runs. After aligning the reads against the 221 SILVA_132 reference database using BLAST, 47,100 unique features with a frequency greater 222 two were obtained.

223

224

225

The faecal microbiome composition varies significantly between sympatric wild rabbits and hares, and between individual rabbits

226

We conducted alpha and beta diversity analyses to assess the species richness, evenness, and

227 composition both within individual animals (alpha diversity) and between rabbits and hares (beta

228 diversity). The richness and evenness of bacterial species in individual animals was not

229 significantly different between rabbits and hares, as measured by the Shannon index and

230

231 Kruskal-Wallis test (Fig. S1). However, the beta diversity between rabbit and hare faecal samples differed significantly $(\mathrm{p}=0.01$ as measured by Bray-Curtis dissimilarity matrix and

232

233 PERMANOVA) (Fig. 1). We also observed considerable variance in faecal microbial beta

234

235

236

237

238

239

240

241

242

243

244

245

246

247

248

249

250

251

252

253

254

255

256

257 diversity between individual rabbits (Fig. 1). In contrast, the hare faecal samples examined all had a similar bacterial composition with relatively low variance between individuals. We observed these effects in both the Illumina and Nanopore $16 \mathrm{~S}$ sequencing data sets (Fig. 1). We did not observe clear correlation between bacterial diversity and sex, reproductive status, or season of sample collection, although our statistical power was low due to the relatively small sample size. Although there were more pregnant and/or lactating rabbits than hares, which may lead to bias in the observed bacterial diversity, this difference was not statistically significant (Fisher's exact test, $\mathrm{p}=0.14$ ). Similarly, the variances of bodyweight for each population were not significantly different (F-test, $\mathrm{p}=0.14)$.

Taxonomic classification of both the Illumina and Nanopore sequencing data sets identified Firmicutes and, to a lesser extent Bacteroidetes, to be the two dominant phyla in both hare and rabbit faecal samples (Fig. 2). On average, Firmicutes and Bacteroidetes together comprised more than $85 \%$ of the faecal microbiome in hares and rabbits and across both platforms. Hare faecal samples contained a significantly higher ratio of Firmicutes to Bacteroidetes compared to rabbit faecal samples ( $\mathrm{p}=0.015$ as measured by Student's t-test). In both hare and rabbit faecal samples, Firmicutes were predominantly represented by the families Ruminococcaceae, Christensenellaceae, Lachnospiraceae (genus Roseburia), Eubacteriaceae, and Erysipelotrichaceaea, while Bacteroidetes were predominantly represented by the families Rikenellaceae and Barnesiellaceae (Fig. 3, Fig. S2). Within the Firmicutes and Bacteroidetes, the families Marinifilaceae (genera Odoribacter and Butryicimonas), Tannerellaceae (genus Parabacteroides), and Veillonellaceae were more abundant in hares compared to rabbits, while the families Clostridiaceae 1, Enterococcaceae, Planococcaceae, Lactobacillaceae, Peptostreptococcaceae, and Bacillaceae were more abundant in rabbits, although not all families were present in all rabbits (Fig. 3, Fig. S2). Phylum Tenericutes (genus Anaeroplasmataceae) 
258

259

260

261

262

263

264

265

266

267

268

269

270

271

272

273

274

275

276

277

278

279

280

281

282

283

284

285

286

287

288

289

290

291

292

293

294

295

296

297 was present at a significantly higher relative frequency in rabbit faecal samples $(\mathrm{p}<0.01$ as measured by Student's t-test). Phylum Verrucomicrobia (genus Akkermansia) was identified only in rabbit samples and not in hare samples, while phyla Lentisphaerae (genus Victivallis) and Synergistetes (genus Pyramidobacter) were identified only in hare samples. Other phyla such as Proteobacteria (genus Sutterella in both hares and rabbits, family Desulfovibrionaceae in hares only, and genus Enterobacter in rabbits only), Actinobacteria (genus Eggerthellaceae), and Patescibacteria (family Saccharimonadaceae) were present at low abundance (0.5-3\%) in both rabbit and hare samples (Fig. 2, Fig. 3, Fig. S2).

We were also able to perform taxonomic classification of Nanopore sequencing data to the species level, due to the longer read lengths associated with this sequencing technology. There was considerable variation in bacterial species between individual faecal samples and many reads remained unassigned at the species level. However, Ruminococcus albus clearly dominated the hare gastrointestinal microbiome, being present in all hare faecal samples at a mean relative frequency of $11.4 \%$ (Table 1). This bacterial species was also consistently detected in rabbit faeces, but at a much lower relative frequency (1.5\%). Of the ten most abundant individual species, hare faeces were dominated by Ruminococcus sp, Bacteroides sp, Alistipes putredinis, and Roseburia hominis; in contrast, rabbit faeces were dominated by several Clostridium and Paraclostridium species, as well as Paeniclostridium sordellii, Ruminococcus albus, and Akkermansia glycaniphila (Table 1).

The NTC microbiome comprised the phyla Proteobacteria and Bacteroidetes at relative frequencies of $99 \%$ and $1 \%$, respectively, from a total of 2158 raw reads (Fig. S3). When analysing the ROCs, there was obvious genomic DNA contamination of both ROCs, with several phyla shared between faecal samples and ROCs (Fig. S3). Additional bacterial phyla were also detected only in ROCs, likely comprising the "reagent microbiome", including Plantomycetes, Dependentiae, Chloroflexi, and Chlamydiae. The total number of raw reads in the rabbit ROC was 6279 (compared to the mean 501,908 reads/sample), while contamination was more pronounced in the hare ROC, which produced 265,118 raw reads. Since the phyla-level distribution of reads in the hare ROC strongly reflected that seen in all hare samples, it was assumed that this contamination would not grossly alter the results, particularly because we were not dealing with low-biomass samples.

\section{Geography alters the faecal microbiome of wild hares}

We then compared the faecal microbiome of Australian wild hares to that of wild hares in their native range of Europe (sampled and sequenced in a previous study (24)) to investigate the influence of geography on the gastrointestinal microbiome. Strikingly, phylum Spirochaetes was the third most abundant phylum in faecal samples of European origin (Fig. 4), yet it was only present at very low abundance from Australian hares and rabbits. Furthermore, phylum Patescibacteria was below our limit of detection $(<0.5 \%$ relative abundance) in European hare 
298

299

300

301

302

303

304

305

306

307

308

309

310

311

312

313

314

315

316

317

\section{8}

319

320

321

322

323

324

325

326

327

328

329

330

331

332

333

334

335

336

337

samples, while in Australian hare samples it was present at a higher relative abundance (1.3\%). Other phyla were detected in both European and Australian hare samples at similar relative abundances (Fig. 2 and 4).

\section{Nanopore and Illumina sequencing platforms reveal a similar faecal microbiome independent of platform}

We found a strong correlation (as measured by Pearson correlation coefficient) between Illumina and Nanopore sequencing results at the phylum $(\mathrm{r}=0.945, \mathrm{p}<0.001)$, family $(\mathrm{r}=0.947, \mathrm{p}<$ 0.001 ), and genus ( $r=0.923, p=<0.001)$ levels (Fig. 2, Fig. 3, Fig. S2). Most notably, phyla Actinobacteria and Synergistetes were present at a higher relative abundance in the Illumina dataset, while phyla Tenericutes, Lentisphaerae, and to a lesser extent Cyanobacteria, were present at higher abundance in the Nanopore dataset (Fig. 2). At the family level, Thermoanaerobacteraceae, Bacteroidaceae, Clostridiales vadinBB60 group, and Atopobiaceae were detected in the Illumina but not the Nanopore data, while Muribaculaceae and Flavobacteriaceae were identified only in the Nanopore data (Fig. 3, Fig. S2). Even at the genus level there was generally good agreement between datasets (Fig. 3). However, genera Butyricimonas, Mailhella, Ruminococcus 2, Erysipelatoclostridium, and Sutterella were only detected in Illumina data and Parasutterella, Schwartzia, an unassigned Victivallaceae, and the Ruminococcaceae NK4A214 group were only identified in the Nanopore data.

\section{Discussion}

To date, studies investigating the gastrointestinal microbial diversity of lagomorphs have been largely limited to domestic production rabbits in either Europe or China (14-17, 19. Currently, two studies have focussed on wild lagomorphs; the first investigating wild rabbits in Wales (23) and the second examining European brown hares in their native European home-range (24). We are interested in understanding why rabbits, an invasive pest species in Australia, were able to rapidly colonise over two thirds of the continent, an area 25 times the size of Britain, within 50 years. In contrast, populations of European brown hares, also a non-native species, remained relatively stable, despite both species occupying a similar dietary niche. Differences in colonising potential of these two species are likely multifactorial, for example, involving differences in behaviour, physiology, and dietary preferences. Comparative analysis of the gastrointestinal microbiome of these two species is one method that can be used to investigate host differences, which may in turn reveal clues to the differences in diet and potentially environmental impacts that each species has. A secondary aim was to use this study as an opportunity to compare short-read (Illumina) and long-read (Nanopore) sequencing technologies for microbial diversity investigations.

We estimated the gastrointestinal microbiome from faecal samples collected from nine wild European brown hares and twelve European rabbits living sympatrically in their non-native 
338

339

340

341

342

343

344

345

346

347

348

349

350

351

352

353

354

355

356

357

358

359

360

361

362

363

364

365

366

367

368

369

370

371

372

373

374

375

376

377

range in Australia. Rabbits had significantly different faecal microbiome compositions compared to hares and showed greater variability in bacterial composition between individuals. Species richness is crucial for imparting resilience to microbial communities, facilitating rapid and effective adaptation to new environmental conditions (48). Species-rich communities can better resist invading pathogens and decreased microbial diversity in humans has been linked to a range of pathologies (48). Although individual hares and rabbits had similar species richness in their faecal microbiomes, at a population level, we found that rabbits varied widely in their species compositions compared to hares. It is interesting to speculate that this may allow for subsets of the population to rapidly expand into new environments. Whether this is an intrinsic feature of Oryctolagus cuniculus or whether this is due to their larger population remains to be determined. In contrast, hares may face more of a 'microbiome bottleneck', which may restrict their ability to adapt to new environments or diets. Hares are known to be more selective foragers than rabbits, which are considered more generalist (7). The observed diversity in the faecal microbiome composition of wild rabbits may be a consequence of highly variable individual dietary preferences, or alternatively, a more diverse faecal microbiome may indeed permit rabbits to consume feeds that hares cannot digest.

The faecal microbiomes of both host species were dominated by the phyla Firmicutes and Bacteroidetes, as is typical for other vertebrate species (49). However, the ratio of Firmicutes to Bacteroidetes varied, being markedly higher in rabbits compared to hares. At the genus level, hare faeces were dominated by Ruminococcus and Bacteroides species, while rabbit faeces were dominated by Clostridium and Paraclostridium species. In humans, an increased Firmicutes to Bacteroidetes ratio has notoriously been associated with age, diet, and obesity (50). A higher abundance of Bacteroidetes (genus Bacteroides) has been linked to consumption of diets rich in protein and fat in humans (51), and indeed, hares are known to prefer diets rich in crude fat and protein (52). Despite having a higher daily digestible nitrogen intake, hares tend to have less efficient protein digestion compared to rabbits, potentially due to the absence of key microbes in their gastrointestinal tract (53). Another apparent difference in the faecal microbiomes of these species was the presence of phylum Verrumicrobia in rabbits. This phylum is dominated by bacteria from the genus Akkermansia, which are noted to be positively influenced by dietary polyphenols $(54,55)$. Polyphenols are metabolised by intestinal bacteria to generate short-chain fatty acids (SCFAs), and previous studies have demonstrated that rabbits produce a higher concentration of SCFAs than hares, with a higher ratio of butyrate to propionate (56-58). Again, it is unknown whether the Akkermansia detected in rabbits in this study permit the digestion of these polyphenols, influencing the dietary preference of rabbits, or whether the intake of polyphenols supports a detectable Akkermansia population in rabbits.

Phyla Lentisphaerae and Synergistetes were observed only in hare populations and were dominated by the genera Victivallis and Pyramidobacter, respectively. Age and diet appear to be important factors in regulating the abundance of these phyla $(59,60)$. A previous study in pigs 
378 reported a decrease in relative abundance of phyla Lentisphaerae and Synergistetes with aging 379 (59). Furthermore, lower relative abundances of phyla Proteobacteria, Lentisphaerae, and 380 Tenericutes in vervets and humans have been associated with "Western diets" rich in

381

382

383

384

385

386

387

388

389

390

391

392

393

394

395

396

397

398

399

400

401

402

403

404

405

406

407

408

409

410

411

412

413

414

415

416

417

carbohydrates (60). The observed differences in faecal microbiota could also be related to other known differences in digestive physiology between rabbits and hares. For example, hares have a higher gastrointestinal passage rate compared to rabbits, while rabbits retain digesta longer in order to maximise the efficiency of nutrient extraction (53). Rabbits also have a greater ability to digest hemicelluloses and have a higher rate of methanogenesis compared to hares $(56-58,61)$.

We also analysed raw data sequenced in a previous study examining faecal pellets of wild hares in their native range of Europe to estimate the influence of geography on the gut microbiome (24). We observed the phylum Spirochaetes to be the third dominant phyla in the European dataset, in accordance with the original analysis. However, in our Australian dataset only very few reads were associated with this phylum (Table S2). Although bacteria within this phylum can be pathogenic, the Spirochetes identified in European hares were associated with a nonpathogenic genus. The absence of this phylum of bacteria in Australian hares may reflect geographical differences in diet between the populations studied, or loss of this bacterial species after introduction into Australia. For example, the hares sampled in this study had access to tropical and native Australian grasses such as Panicum effusum, Themeda australis, Eragrostis benthamii, Stipa bigeniculata, and Austrostipa scabra, which would not have been available to the hares sampled in the European study. In contrast, hares in Europe have been reported to preferentially graze on cereal crops and grasses such as soybean, beet plants, barley, alfalfa, knotgrass, chickweed, clover, and maize (52). These species were not available to the hares sampled in our Autralian study. Alternatively, different sample handling e.g. different primers used for $16 \mathrm{~S}$ amplification, may possibly have resulted in selective bias against Spirochaetes in our study. Despite these spirochaetes likely being non-pathogenic, it is worth noting that the absence of pathogens of invasive species in their non-native range may contribute to spread and persistence of these alien species in new environments. Since faecal samples in this study were collected from healthy hares and rabbits, no candidate pathogens were investigated here.

An additional aim of this study was to compare the bacterial diversity between two widely used sequencing platforms. The Illumina platform is a popular approach for 16S rRNA sequencing, particularly because of its lower error rate (62). However, the short-read length can make species level identification very challenging, especially between closely related species (62). In contrast, the Oxford Nanopore platform has the ability to sequence very long reads, however, it is prone to a relatively high error rate, again making accurate taxonomic assignment challenging (62). In this study, we observed very similar bacterial diversity at the phylum, family, and genus levels with both sequencing platforms, confirming the suitability of either technology for 16S rRNA studies and further confirming the biological relevance of our findings. The minor observed differences in relative abundances of different phyla are most likely due to PCR-based errors or

Peer) reviewing PDF | (2020:04:47575:1:1:NEW 5 Jun 2020) 
418 bias, since different primer sets were used for each sequencing platform, or bias during 419 sequencing (62). The strong correlation between Illumina and Nanopore data suggests that 420 despite the higher error rate of Nanopore technology, this is not a significant issue for taxonomic 421 classification, at least at the genus level or higher. With the additional benefits of longer reads 422 and species level classification of Nanopore data, this study highlights that there are clear 423 benefits to the use of the Nanopore platform for metagenomics studies.

424

\section{Conclusions}

426

427

In conclusion, we observed notable differences in the microbiome of hares and rabbits living in

428

429 sympatry in their non-native range in Australia. Though these species inhabit the same habitat, their behaviour and dietary preferences clearly influence differences in faecal bacterial composition. The more variable gastrointestinal microbiota of rabbits compared to hares could be a contributing factor in their ability to spread very successfully and establish in new environments. Additionally, the absence of bacteria from the phylum Spirochaetes in Australian compared to European wild hares (where it was observed frequently and often at high relative abundance) demonstrates considerable geographical differences between populations, although whether these spirochetes are beneficial or detrimental to hares was not determined. Future studies correlating different bacterial species in lagomorph microbiomes with specific plant species may provide further insights into the impacts of wild rabbits and hares in Australia.

\section{Acknowledgements}

440 We thank Barry Richardson, Oliver Orgill, and the ACT Parks and Conservation Service rangers 441 of Mulligan's Flat Woodland Sanctuary for their assistance with sampling. We also thank

442 Gabrielle Stalder, Stefanie Wetzels, and Evelyne Mann for sharing their previously published 443 raw data from hares in Europe. We also wish to thank Amanda Padovan and Maria Jenckel for 444 critical revision of the manuscript.

445

\section{References}

447 1. Čuda J, Skálová H, Janovský Z, Pyšek P. 2015. Competition among native and invasive 448 Impatiens species: the roles of environmental factors, population density and life stage. AoB 449 PLANTS 7.

450 2. Shine R. 2010. The ecological impact of invasive cane toads (Bufo marinus) in Australia. 451 Q Rev Biol 85:253-291.

452 3. Stott P. 2003. Use of space by sympatric European hares (Lepus europaeus) and 453 European rabbits (Oryctolagus cuniculus) in Australia. Mamm Biol 68:317-327.

454 4. Zenger KR, Richardson BJ, Vachot-Griffin A-M. 2003. A rapid population expansion 455 retains genetic diversity within European rabbits in Australia. Mol Ecol 12:789-794.

456 5. Bird P, Mutze G, Peacock D, Jennings S. 2012. Damage caused by low-density exotic 457 herbivore populations: The impact of introduced European rabbits on marsupial herbivores and 
458 Allocasuarina and Bursaria seedling survival in Australian coastal shrubland. Biol Invasions 459 14:743-755.

460 6. Department of the Environment and Energy. 2016. Threat abatement plan for 461 competition and land degradation by rabbits.

462 https://www.environment.gov.au/biodiversity/threatened/publications/tap/competition-and-land463 degradation-rabbits-2016. Accessed 16 July 2019.

$4647 . \quad$ Chapuis J. 1990. Comparison of the diets of two sympatric lagomorphs, Lepus europaeus 465 Pallas and Oryctolagus cuniculus. Z Säugetierkunde 55:176-185.

466 8. David LA, Maurice CF, Carmody RN, Gootenberg DB, Button JE, Wolfe BE, Ling AV, 467 Devlin AS, Varma Y, Fischbach MA, Biddinger SB, Dutton RJ, Turnbaugh PJ. 2013. Diet 468 rapidly and reproducibly alters the human gut microbiome. Nature 505:559-563.

469 9. Henderson G, Cox F, Ganesh S, Jonker A, Young W, Global Rumen Census

470 Collaborators, Janssen PH. 2015. Rumen microbial community composition varies with diet and 471 host, but a core microbiome is found across a wide geographical range. Sci Rep 5:14567.

472 10. Clemente Jose C, Ursell Luke K, Parfrey Laura W, Knight R. 2012. The impact of the gut 473 microbiota on human health: An integrative view. Cell 148:1258-1270.

474 11. McKenna P, Hoffmann C, Minkah N, Aye PP, Lackner A, Liu Z, Lozupone CA, Hamady 475 M, Knight R, Bushman FD. 2008. The macaque gut microbiome in health, lentiviral infection, 476 and chronic enterocolitis. PLOS Pathogens 4:e20.

477 12. Turnbaugh PJ, Ley RE, Hamady M, Fraser-Liggett CM, Knight R, Gordon JI. 2007. The 478 human microbiome project. Nature 449:804-810.

479 13. Alcock J, Maley CC, Aktipis CA. 2014. Is eating behavior manipulated by the 480 gastrointestinal microbiota? Evolutionary pressures and potential mechanisms. BioEssays $481 \quad 36: 940-949$.

482 14. Eshar D, Weese JS. 2014. Molecular analysis of the microbiota in hard feces from 483 healthy rabbits (Oryctolagus cuniculus) medicated with long term oral meloxicam. BMC Vet 484 Res 10:62.

485 15. Zeng B, Han S, Wang P, Wen B, Jian W, Guo W, Yu Z, Du D, Fu X, Kong F, Yang M, 486 Si X, Zhao J, Li Y. 2015. The bacterial communities associated with fecal types and body weight 487 of rex rabbits. Sci Rep 5:9342-9342.

488 16. Kylie J, Weese JS, Turner PV. 2018. Comparison of the fecal microbiota of domestic 489 commercial meat, laboratory, companion, and shelter rabbits (Oryctolagus cuniculi). BMC Vet 490 Res 14:143.

491 17. Velasco-Galilea M, Piles M, Viñas M, Rafel O, González-Rodríguez O, Guivernau M, 492 Sánchez JP. 2018. Rabbit microbiota changes throughout the intestinal tract. Front Microbiol 493 9:2144.

494 18. Reddivari L, Veeramachaneni DNR, Walters WA, Lozupone C, Palmer J, Hewage MKK, 495 Bhatnagar R, Amir A, Kennett MJ, Knight R, Vanamala JKP. 2017. Perinatal bisphenol A 496 exposure induces chronic inflammation in rabbit offspring via modulation of gut bacteria and 497 their metabolites. mSystems 2:e00093-17. 
498 19. Xing Y, Liu J, Lu F, Wang L, Li Y, Ouyang C. 2019. Dynamic distribution of gallbladder 499 microbiota in rabbit at different ages and health states. PLOS ONE 14:e0211828.

500 20. Dabbou S, Ferrocino I, Kovitvadhi A, Bergagna S, Dezzuto D, Schiavone A, Cocolin L, 501 Gai F, Santoro V, Gasco L. 2019. Bilberry pomace in rabbit nutrition: effects on growth 502 performance, apparent digestibility, caecal traits, bacterial community and antioxidant status. 503 Animal 13:53-63.

504 21. Xing Y-W, Lei G-T, Wu Q-H, Jiang Y, Huang M-X. 2019. Procyanidin B2 protects 505 against diet-induced obesity and non-alcoholic fatty liver disease via the modulation of the gut 506 microbiota in rabbits. World J Gastroentero 25:955-966.

507 22. O' Donnell MM, Harris HMB, Ross RP, O'Toole PW. 2017. Core fecal microbiota of 508 domesticated herbivorous ruminant, hindgut fermenters, and monogastric animals.

509 MicrobiologyOpen 6:e00509.

510 23. Crowley EJ, King JM, Wilkinson T, Worgan HJ, Huson KM, Rose MT, McEwan NR.

511 2017. Comparison of the microbial population in rabbits and guinea pigs by next generation 512 sequencing. PLOS ONE 12:e0165779.

513 24. Stalder GL, Pinior B, Zwirzitz B, Loncaric I, Jakupović D, Vetter SG, Smith S, Posautz 514 A, Hoelzl F, Wagner M, Hoffmann D, Kübber-Heiss A, Mann E. 2019. Gut microbiota of the 515 European brown hare (Lepus europaeus). Sci Rep 9:2738.

516 25. Redford KH, Segre JA, Salafsky N, del Rio CM, McAloose D. 2012. Conservation and 517 the microbiome. Conserv Biol 26:195-197.

518 26. Gong B, Cao H, Peng C, Perčulija V, Tong G, Fang H, Wei X, Ouyang S. 2019. High519 throughput sequencing and analysis of microbial communities in the mangrove swamps along 520 the coast of Beibu Gulf in Guangxi, China. Sci Rep 9:9377.

521 27. Wei Y-J, Wu Y, Yan Y-Z, Zou W, Xue J, Ma W-R, Wang W, Tian G, Wang L-Y. 2018. 522 High-throughput sequencing of microbial community diversity in soil, grapes, leaves, grape juice 523 and wine of grapevine from China. PLOS ONE 13:e0193097.

524 28. Jackson CR, Randolph KC, Osborn SL, Tyler HL. 2013. Culture dependent and 525 independent analysis of bacterial communities associated with commercial salad leaf vegetables. 526 BMC Microbiol 13:274.

527 29. Stefani FOP, Bell TH, Marchand C, de la Providencia IE, El Yassimi A, St-Arnaud M, 528 Hijri M. 2015. Culture-dependent and -independent methods capture different microbial 529 community fractions in hydrocarbon-contaminated soils. PLOS ONE 10:e0128272.

530 30. Öztürk B, de Jaeger L, Smidt H, Sipkema D. 2013. Culture-dependent and independent 531 approaches for identifying novel halogenases encoded by Crambe crambe (marine sponge) 532 microbiota. Sci Rep 3:2780.

533 31. Adewumi G, Oguntoyinbo F, Keisam S, Jeyaram K. 2013. Combination of culture534 independent and culture-dependent molecular methods for the determination of bacterial 535 community of iru, a fermented Parkia biglobosa seeds. Front Microbiol 3:436. 
536 32. Ranjan R, Rani A, Metwally A, McGee HS, Perkins DL. 2016. Analysis of the 537 microbiome: Advantages of whole genome shotgun versus $16 \mathrm{~S}$ amplicon sequencing. Biochem 538 Bioph Res Co 469:967-977.

539 33. Schadt EE, Turner S, Kasarskis A. 2010. A window into third-generation sequencing.

540 Hum Mol Genet 19:227-240.

541 34. Lee H, Gurtowski J, Yoo S, Nattestad M, Marcus S, Goodwin S, Richard McCombie W, 542 Schatz MC. 2016. Third-generation sequencing and the future of genomics. bioRxiv

543 doi:10.1101/048603:048603.

544 35. Shin J, Lee S, Go M-J, Lee SY, Kim SC, Lee C-H, Cho B-K. 2016. Analysis of the

545 mouse gut microbiome using full-length 16S rRNA amplicon sequencing. Sci Rep 6:29681.

546 36. Illumina. 2013. 16s metagenomic sequencing library preparation.

547 http://sapac.support.illumina.com/content/dam/illumina-

548 support/documents/documentation/chemistry documentation/16s/16s-metagenomic-library-prep549 guide-15044223-b.pdf. Accessed 5 July 2019.

550 37. Bolyen E, Rideout JR, Dillon MR, Bokulich NA, Abnet C, Al-Ghalith GA, Alexander H,

551 Alm EJ, Arumugam M, Asnicar F. 2019. Reproducible, interactive, scalable and extensible

552 microbiome data science using QIIME 2. Nat Biotechnol 37:852-857.

553 38. Callahan BJ, McMurdie PJ, Rosen MJ, Han AW, Johnson AJA, Holmes SP. 2016.

554 DADA2: High-resolution sample inference from Illumina amplicon data. Nat Methods 13:581-

555583.

556 39. Bokulich NA, Kaehler BD, Rideout JR, Dillon M, Bolyen E, Knight R, Huttley GA,

557 Gregory Caporaso J. 2018. Optimizing taxonomic classification of marker-gene amplicon

558 sequences with QIIME 2's q2-feature-classifier plugin. Microbiome 6:90.

559 40. Camacho C, Coulouris G, Avagyan V, Ma N, Papadopoulos J, Bealer K, Madden TL.

560 2009. BLAST+: architecture and applications. BMC Bioinformatics 10:421.

561 41. Quast C, Pruesse E, Yilmaz P, Gerken J, Schweer T, Yarza P, Peplies J, Glöckner FO.

562 2013. The SILVA ribosomal RNA gene database project: improved data processing and web-

563 based tools. Nucleic Acids Res 41:590-596.

564 42. Faith DP, Minchin PR, Belbin L. 1987. Compositional dissimilarity as a robust measure 565 of ecological distance. Vegetatio 69:57-68.

566 43. Anderson MJ. 2001. A new method for non-parametric multivariate analysis of variance.

567 Austral Ecology 26:32-46.

568 44. Weisburg WG, Barns SM, Pelletier DA, Lane DJ. 1991. 16S ribosomal DNA

569 amplification for phylogenetic study. J Bacteriol 173:697-703.

570 45. Hu Y, Schwessinger B. 2018. Amplicon sequencing using MinION optimized from 1D

571 native barcoding genomic DNA. protocols io doi:dx.doi.org/10.17504/protocols.io.mhkc34w.

572 46. Wick RR, Judd LM, Holt KE. 2018. Deepbinner: Demultiplexing barcoded Oxford

573 Nanopore reads with deep convolutional neural networks. PLOS Comp Biol 14:e1006583.

574 47. Kruskal WH, Wallis WA. 1952. Use of Ranks in One-Criterion Variance Analysis.

575 Journal of the American Statistical Association 47:583-621. 
576 48. Lozupone CA, Stombaugh JI, Gordon JI, Jansson JK, Knight R. 2012. Diversity, stability 577 and resilience of the human gut microbiota. Nature 489:220-230.

578 49. Eckburg PB, Bik EM, Bernstein CN, Purdom E, Dethlefsen L, Sargent M, Gill SR, 579 Nelson KE, Relman DA. 2005. Diversity of the human intestinal microbial flora. Science 580 308:1635-1638.

581 50. Ley RE, Turnbaugh PJ, Klein S, Gordon JI. 2006. Human gut microbes associated with 582 obesity. Nature 444:1022-1023.

583 51. Wu GD, Chen J, Hoffmann C, Bittinger K, Chen Y-Y, Keilbaugh SA, Bewtra M, Knights 584 D, Walters WA, Knight R, Sinha R, Gilroy E, Gupta K, Baldassano R, Nessel L, Li H, Bushman 585 FD, Lewis JD. 2011. Linking long-term dietary patterns with gut microbial enterotypes. Science 586 334:105-108.

587 52. Schai-Braun SC, Reichlin TS, Ruf T, Klansek E, Tataruch F, Arnold W, Hackländer K. 588 2015. The European hare (Lepus europaeus): a picky herbivore searching for plant parts rich in 589 fat. PLOS ONE 10:e0134278.

590 53. Kuijper D, Van Wieren S, Bakker J. 2004. Digestive strategies in two sympatrically 591 occurring lagomorphs. J Zool 264:171-178.

$59254 . \quad$ Roopchand DE, Carmody RN, Kuhn P, Moskal K, Rojas-Silva P, Turnbaugh PJ, Raskin

593 I. 2015. Dietary polyphenols promote growth of the gut bacterium Akkermansia muciniphila and 594 attenuate high-fat diet-induced metabolic syndrome. Diabetes 64:2847-2858.

595 55. Anhê FF, Pilon G, Roy D, Desjardins Y, Levy E, Marette A. 2016. Triggering

596 Akkermansia with dietary polyphenols: A new weapon to combat the metabolic syndrome? Gut 597 Microbes 7:146-153.

598 56. Miśta D, Króliczewska B, Marounek M, Pecka E, Zawadzki W, Nicpoń J. 2015. In vitro 599 study and comparison of caecal methanogenesis and fermentation pattern in the brown hare 600 (Lepus europaeus) and domestic rabbit (Oryctolagus cuniculus). PLOS ONE 10:e0117117. 601 57. Marounek M, Brezina P, Baran M. 2000. Fermentation of carbohydrates and yield of 602 microbial protein in mixed cultures of rabbit caecal microorganisms. Arch Tierernahr 53:241603252.

604 58. Miśta D, Króliczewska B, Pecka-Kiełb E, Piekarska J, Marounek M, Zawadzki W. 2018. 605 Comparative in vitro study of caecal microbial activity in brown hares and domestic rabbits 606 which were offered the same diet. Mammal Res 63:285-296.

607 59. Niu Q, Li P, Hao S, Zhang Y, Kim SW, Li H, Ma X, Gao S, He L, Wu W, Huang X, Hua 608 J, Zhou B, Huang R. 2015. Dynamic distribution of the gut microbiota and the relationship with 609 apparent crude fiber digestibility and growth stages in pigs. Sci Rep 5:9938.

610 60. Amato KR, Yeoman CJ, Cerda G, A. Schmitt C, Cramer JD, Miller MEB, Gomez A, R. 611 Turner T, Wilson BA, Stumpf RM, Nelson KE, White BA, Knight R, Leigh SR. 2015. Variable 612 responses of human and non-human primate gut microbiomes to a Western diet. Microbiome 613 3:53. 
614 61. Stott P. 2008. Comparisons of digestive function between the European hare (Lepus 615 europaeus) and the European rabbit (Oryctolagus cuniculus): Mastication, gut passage, and 616 digestibility. Mamm Biol 73:276-286.

617 62. Pollock J, Glendinning L, Wisedchanwet T, Watson M. 2018. The madness of

618 microbiome: Attempting to find consensus "best practice" for 16S microbiome studies. Appl 619 Environ Microbiol 84: e02627. 


\section{Figure Legends}

621

622

623

624

625

626

627

628

629

630

631

632

633

634

635

636

637

638

639

640

641

642

643

644

645

646

647

648

649

650

651

652

653

654

655

656

657

658

Figure 1: The composition of faecal microbial communities varied significantly between wild rabbits and hares and between individual rabbits. 16S rRNA profiling was conducted on wild hare and rabbit faecal samples using either (A) Illumina, or (B) Nanopore sequencing platforms. Principal coordinates analysis of beta diversity using Bray-Curtis dissimilarity (as calculated in QIIME2) was used to compare the microbial species compositions. Significance was assessed using PERMANOVA. Numbers refer to individual animal identifiers as described in Table S1. Stars symbolises pregnant and lactating females, open circles symbolise lactating females, and closed circles symbolise non-pregnant and non-lactating animals.

Figure 2: The faecal microbiomes of wild rabbits and hares were distinct at phylum level. Taxonomic classification of 16S rRNA sequences from (A) Illumina, and (B) Nanopore sequencing platforms was performed by alignment to the SILVA_132 reference database using BLAST+ within QIIME2 for Illumina data or BLASTn for Nanopore data. The relative frequency of reads assigned to bacterial phyla present at an abundance greater than $0.5 \%$ were plotted for each sample.

Figure 3: Faecal microbial diversity of Australian wild hares and rabbits at the family level identified unique profiles for wild hares and rabbits. Taxonomic classification of $16 \mathrm{~S}$ rRNA sequences from (A) Illumina, and (B) Nanopore sequencing platforms was performed by alignment to the SILVA_132 reference database using BLAST+ within QIIME2 for Illumina data or BLASTn for Nanopore data. The frequency of reads mapping to bacterial families for individual samples are shown in a heatmap on a $\log 10$ scale. Family and genera names are highlighted according to phylum. Shapes have been added to selected phyla for improved accessibility. Rows are clustered based on features identified across all samples. Bacterial families present at a frequency less than $0.5 \%$ are not included.

Figure 4: Faecal microbial diversity of wild hares in their native range of Europe shows Spirochetes to be the third most abundant bacterial phyla. Raw Illumina sequencing data were obtained from a previous study examining the $16 \mathrm{~S}$ rRNA diversity in faecal pellets of European brown hares in Europe (24). Reads were processed and classified in parallel with sequencing data from Australia hare faecal samples, using BLAST+ as implemented in QIIME2 against the SILVA_132 reference database. The relative frequency of reads assigned to bacterial phyla present at an abundance greater than $0.5 \%$ were plotted for each sample. Australian hare data presented here are repeated from Figure 2 for ease of comparison.

Supplementary Figure 1: Shannon diversity analysis of wild rabbits and hares. The richness and evenness of bacterial species in individual animals (alpha diversity) was estimated using the 
659 Shannon index calculated in QIIME2, and was compared between rabbits and hares using the 660 Kruskal-Wallis test as implemented in the R package 'ggpubr'.

661

662 Supplementary Figure 2: Illumina and Nanopore sequencing platforms produced very 663 similar distributions of bacterial families in wild hare and rabbit faecal samples. Taxonomic 664 classification of 16S rRNA sequences from (A) Illumina, and (B) Nanopore sequencing 665 platforms was performed by alignment to the SILVA_132 reference database using BLAST+ 666 within QIIME2 for Illumina data or BLASTn for Nanopore data. Results were combined for 667 each host species (i.e. hare or rabbit) for each platform. Bacterial families present at a relative 668 frequency less than $0.5 \%$ are not included.

669 Supplementary Figure 3: Taxonomic bar plot at phyla rank showing bacterial composition 671 across ROC and NTC samples. Reagent-only controls (ROCs) were extracted and processed in 672 parallel with genomic DNA from faecal pellets. The no template control (NTC) was included in 673 16S rRNA PCRs and subsequently processed in parallel with samples. Samples were sequenced 674 using the Illumina platform. Taxonomic classification of reads was performed using BLAST + as 675 implemented in QIIME2 against the SILVA_132 reference database. Bacterial phyla present at a 676 relative frequency less than $0.5 \%$ are not included. 


\section{Table 1 (on next page)}

Top ten bacterial species identified in hare and rabbit faecal samples using Nanopore sequencing 
1 Table 1: Top ten bacterial species identified in hare and rabbit faecal samples using Nanopore 2 sequencing

\begin{tabular}{|c|c|c|c|c|}
\hline Phylum & Family & Genus & Species & $\begin{array}{l}\text { Mean relative } \\
\text { abundance }(\%)\end{array}$ \\
\hline \multicolumn{5}{|l|}{ Hares } \\
\hline Firmicutes & Ruminococcaceae & Ruminococcus & albus & 11.360 \\
\hline Bacteroidetes & Bacteroidaceae & Bacteroides & uniformis & 3.712 \\
\hline Firmicutes & Ruminococcaceae & Ruminococcus & champanellensis & 3.251 \\
\hline Bacteroidetes & Bacteroidaceae & Bacteroides & sartorii & 1.578 \\
\hline Firmicutes & Ruminococcaceae & Ruminococcus & flavefaciens & 1.546 \\
\hline Bacteroidetes & Bacteroidaceae & Bacteroides & mediterraneensis & 1.471 \\
\hline Bacteroidetes & Bacteroidaceae & Bacteroides & eggerthii & 1.322 \\
\hline Bacteroidetes & Bacteroidaceae & Bacteroides & caecigallinarum & 1.068 \\
\hline Bacteroidetes & Rikenellaceae & Alistipes & putredinis & 1.066 \\
\hline Firmicutes & Lachnospiraceae & Roseburia & hominis & 0.980 \\
\hline \multicolumn{5}{|l|}{ Rabbits } \\
\hline Firmicutes & Clostridiaceae & Clostridium & baratii & 4.239 \\
\hline Firmicutes & Clostridiaceae & Clostridium & moniliforme & 2.837 \\
\hline Firmicutes & Peptostreptococcaceae & Paeniclostridium & sordellii & 2.258 \\
\hline Firmicutes & Clostridiaceae & Clostridium & paraputrificum & 1.710 \\
\hline Firmicutes & Peptostreptococcaceae & Paraclostridium & bifermentans & 1.619 \\
\hline Firmicutes & Clostridiaceae & Clostridium & senegalense & 1.541 \\
\hline Firmicutes & Ruminococcacaea & Ruminococcus & albus & 1.520 \\
\hline Firmicutes & Clostridiaceae & Clostridium & argentinense & 0.873 \\
\hline Firmicutes & Peptostreptococcaceae & Paraclostridium & benzoelyticum & 0.764 \\
\hline Verrucomicrobia & Akkermansiaceae & Akkermansia & glycaniphila & 0.728 \\
\hline
\end{tabular}




\section{Figure 1}

The composition of faecal microbial communities varied significantly between wild rabbits and hares and between individual rabbits.

16S rRNA profiling was conducted on wild hare and rabbit faecal samples using either (A)

Illumina, or (B) Nanopore sequencing platforms. Principal coordinates analysis of beta diversity using Bray-Curtis dissimilarity (as calculated in QIIME2) was used to compare the microbial species compositions. Significance was assessed using PERMANOVA. Numbers refer to individual animal identifiers as described in Table S1. Stars symbolises pregnant and lactating females, open circles symbolise lactating females, and closed circles symbolise nonpregnant and non-lactating animals. 


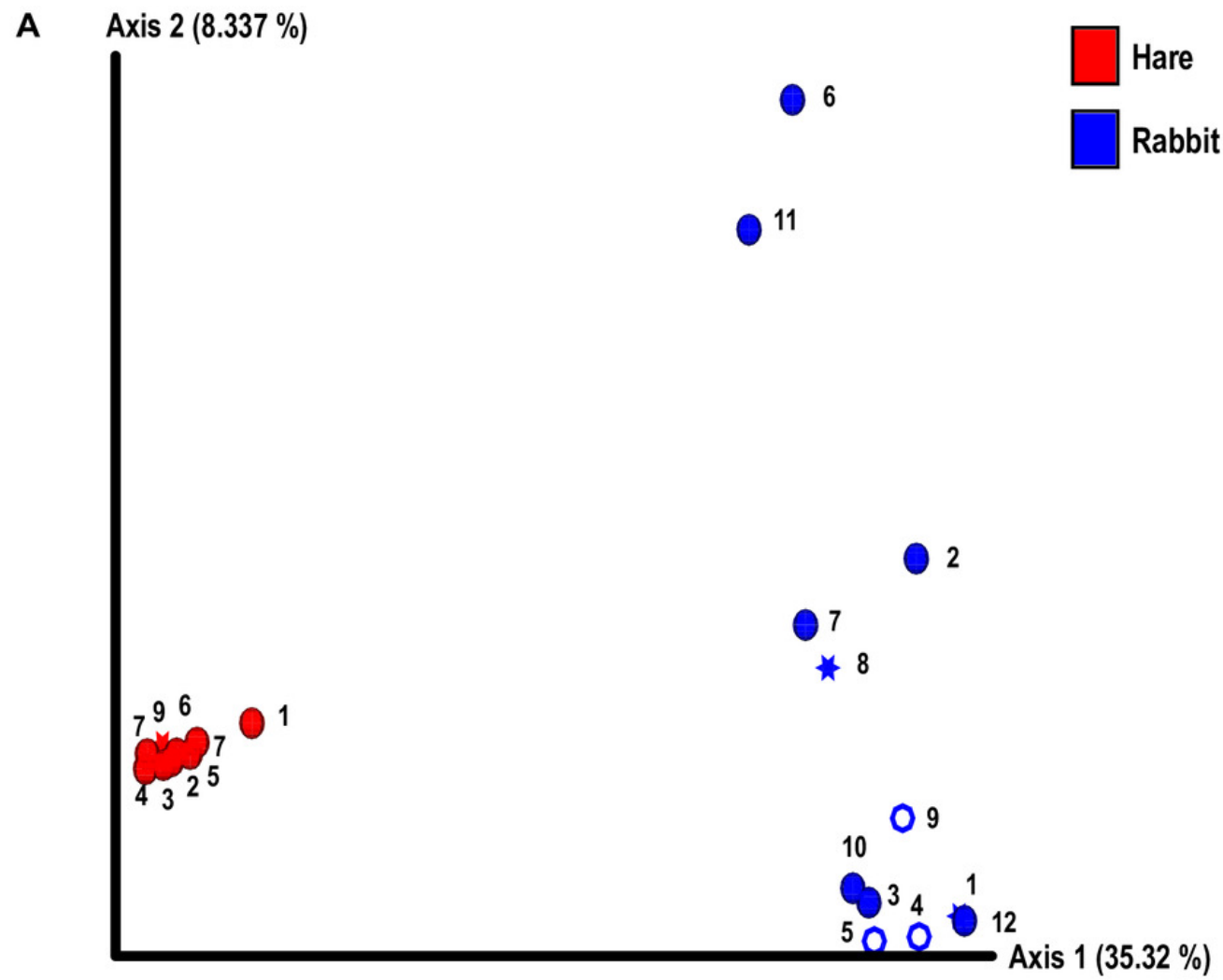

B $\quad$ Axis $2(15.91 \%)$

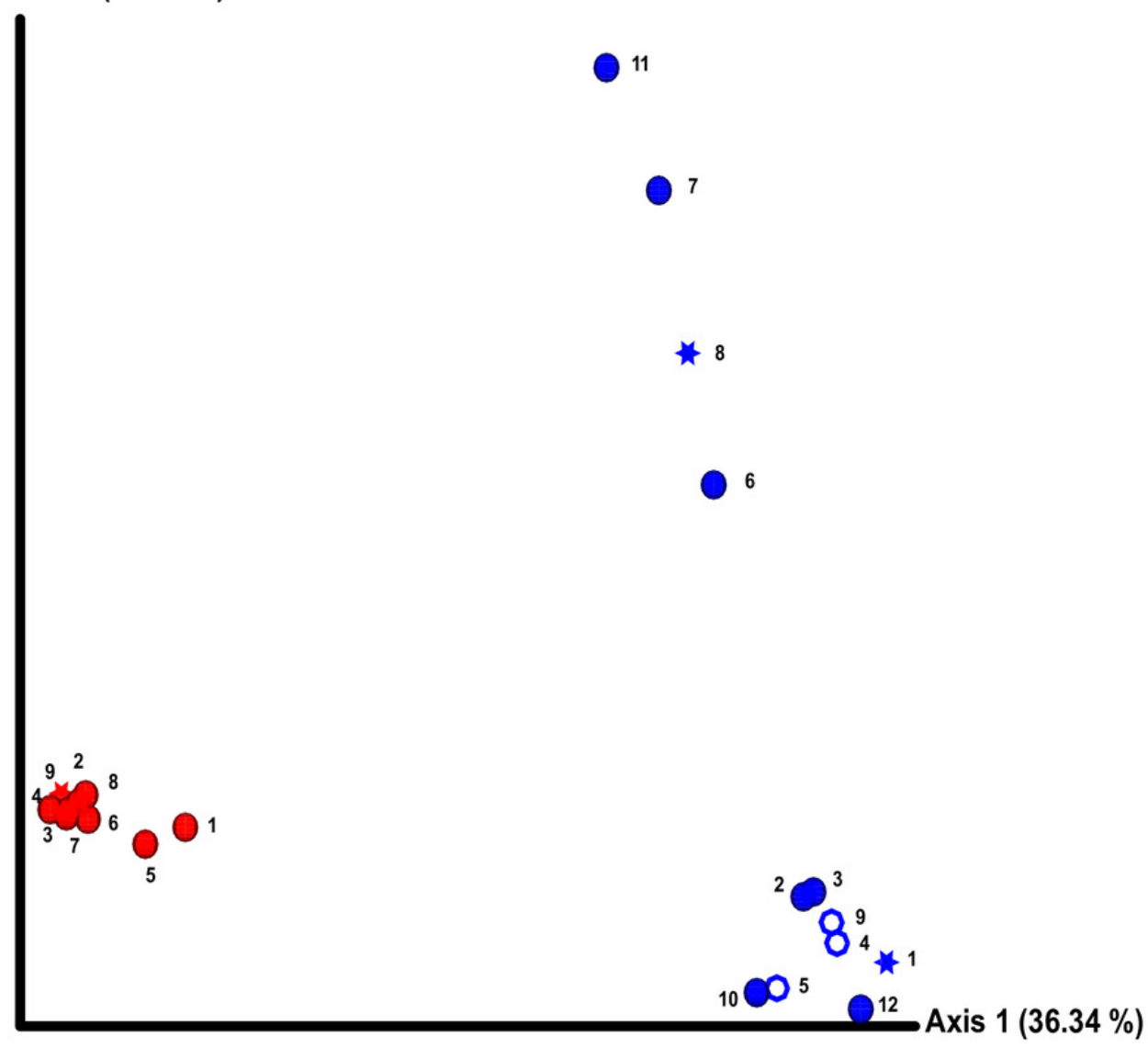




\section{Figure 2}

The faecal microbiomes of wild rabbits and hares were distinct at phylum level.

Taxonomic classification of 165 rRNA sequences from (A) Illumina, and (B) Nanopore sequencing platforms was performed by alignment to the SILVA_132 reference database using BLAST+ within QIIME2 for Illumina data or BLASTn for Nanopore data. The relative frequency of reads assigned to bacterial phyla present at an abundance greater than $0.5 \%$ were plotted for each sample. 


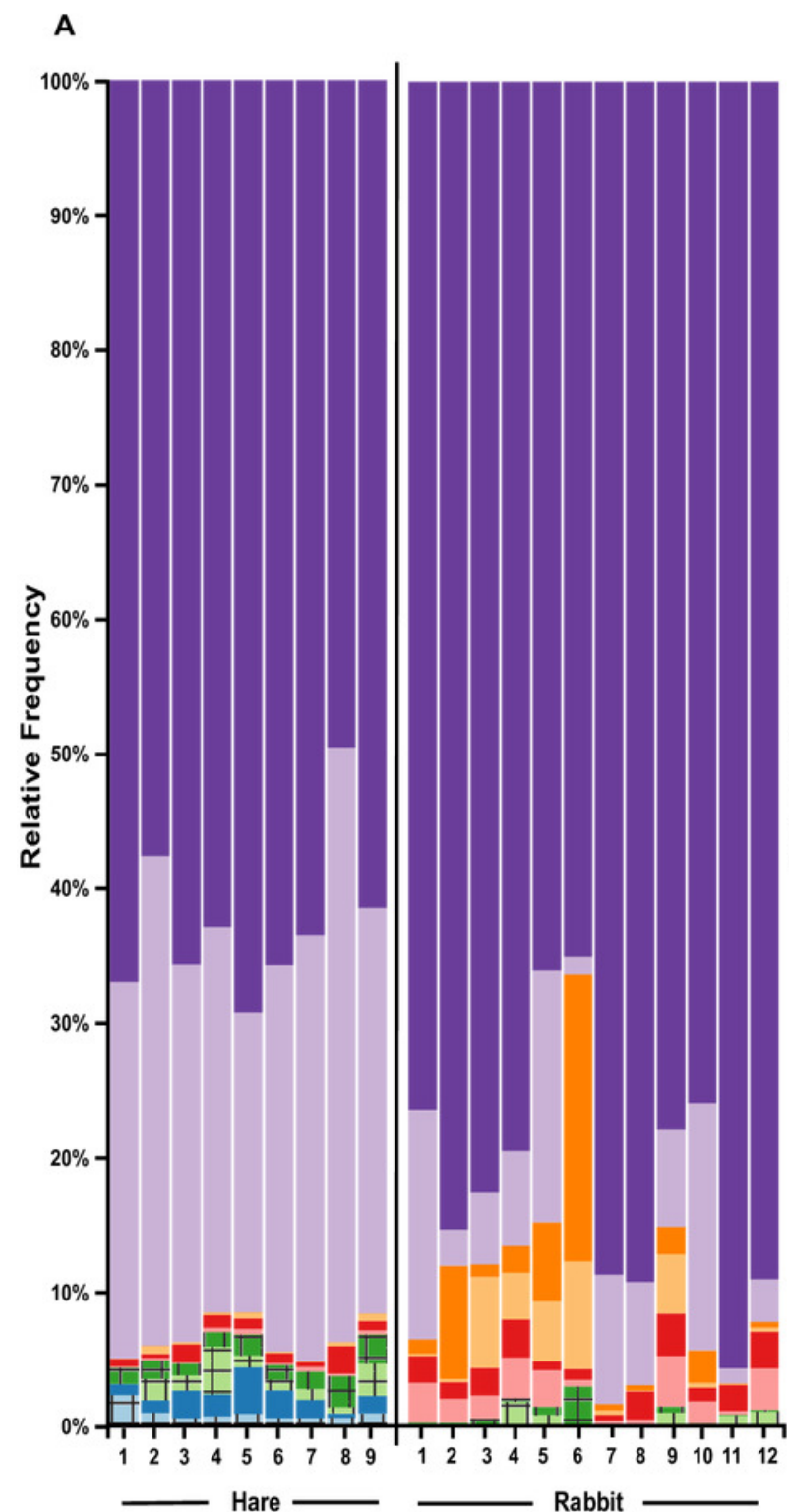

B

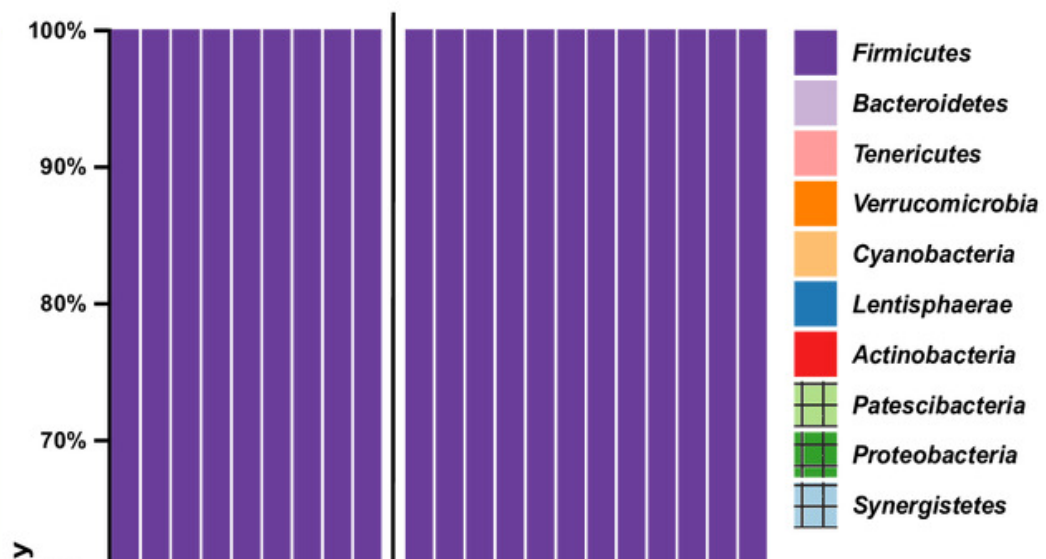




\section{Figure 3}

Faecal microbial diversity of Australian wild hares and rabbits at the family level identified unique profiles for wild hares and rabbits.

Taxonomic classification of 16S rRNA sequences from (A) Illumina, and (B) Nanopore sequencing platforms was performed by alignment to the SILVA_132 reference database using BLAST+ within QIIME2 for Illumina data or BLASTn for Nanopore data. The frequency of reads mapping to bacterial families for individual samples are shown in a heatmap on a $\log 10$ scale. Family and genera names are highlighted according to phylum. Shapes have been added to selected phyla for improved accessibility. Rows are clustered based on features identified across all samples. Bacterial families present at a frequency less than $0.5 \%$ are not included. 
A

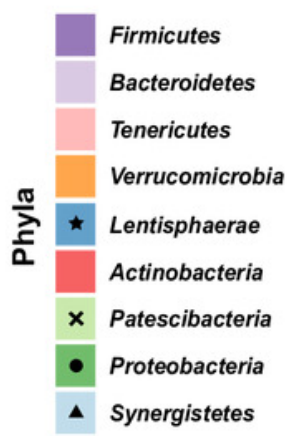

(Genus Clostridium sensu stricto 1) Clostridiaceae (Genus Thermoanaerobacter) Thermoanaerobacteraceae (Genus Akkermansia) Akkermansiaceae (Genus Enterococcus) Enterococcaceae (Genus Rummeliibacillu§ Planococcacea (Genus Lactobacillus) Lactobacillaceae (Genus Anaeroplasma) Anaeroplasmataceae

(Genera Paeniclostridium and Paraclostridium) Peptostreptococcaceae (Genus Bacillus) Bacillaceae

-(Genus Enterobacter) Enterobacteriaceae (Genera Odoribacter and Butyricimonas) Marinifilaceae - (Genera Mailhella and Desulfovibrio) Desulfovibrionaceae (Genus Parabacteroides) Tannerellaceae

$\star$ (Genus Victivallis) Victivallaceae (Genus Unassigned) Veillonellaceae

A (Genus Pyramidobacter) Synergistaceae (Genera Ruminococcus 1 and Ruminococcus 2) Ruminococcacea

(Genus Bacteroides) Bacteroidaceae (Genus Rikenellaceae RC9 gut group) Rikenellaceae (Genus Christensenellaceae R-7 group) Christensenellaceae (Genus Roseburia) Lachnospiraceae (Genus Uncultured bacterium) Clostridiales vadinBB60 group

(Genus Enterorhabdus) Eggerthellaceae (Genus Uncultured bacterium) Eubacteriaceae (Genus Erysipelatoclostridium) Erysipelotrichaceae (Genus Unassigned) Atopobiaceae

(Genus Uncultured bacterium) Barnesiellaceae x(Genus Candidatus Saccharimonas) Saccharimonadaceae

- (Genus Sutterella) Burkholderiaceae

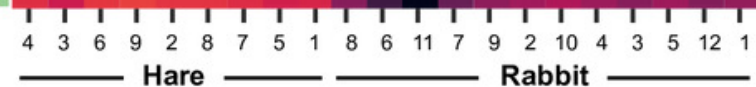

B
(Genera Paeniclostridium and Paraclostridium) Peptostreptococcaceae (Genus Clostridium sensu stricto 1) Clostridiaceae 1 (Genus Rummeliibacillus) planococcaceae

- (Genus Enterobacter) Enterobacteriaceae (Genus Anaeroplasma) Anaeroplasmataceae (Genus Bacillus) Bacillaceae (Genus Lactobacillus) Lactobacillaceae (Genus Enterococcus) Enterococcaceae - (Genus Parasutterella) Burkholderiaceae (Genus Unassigned) Muribaculacea (Genus Uncultured bacterium) Flavobacteriaceae (Genus Parabacteroides) Tannerellaceae

- (Genus Desulfovibrio) Desulfovibrionaceae A (Genus Pyramidobacter) Synergistaceae (Genus Schwartzia) Veillonellaceae $\star$ (Genera Unassigned and Victivallis) Victivallaceae (Genus Odoribacter) Marinifilaceae (Genus Akkermansia) Akkermansiaceae (Genus Uncultured bacterium) Eubacteriaceae (Genus Uncultured bacterium) Erysipelotrichacea

(Genus Uncultured bacterium) Barnesiellaceae (Genus Enterorhabdus) Eggerthellaceae x (Genus Candidatus Saccharimonas) Saccharimonadaceae (Genus Roseburia) Lachnospiraceae (Genus Christensenellaceae R-7 group) Christensenellaceae

(Genus Rikenellaceae RC9 gut group) Rikenellaceae (Genus Clostridium sensu stricto 1) Clostridiaceae 1 (Genera Ruminococcus 1 and Ruminococcaceae NK4A214 group) Ruminococcaceae

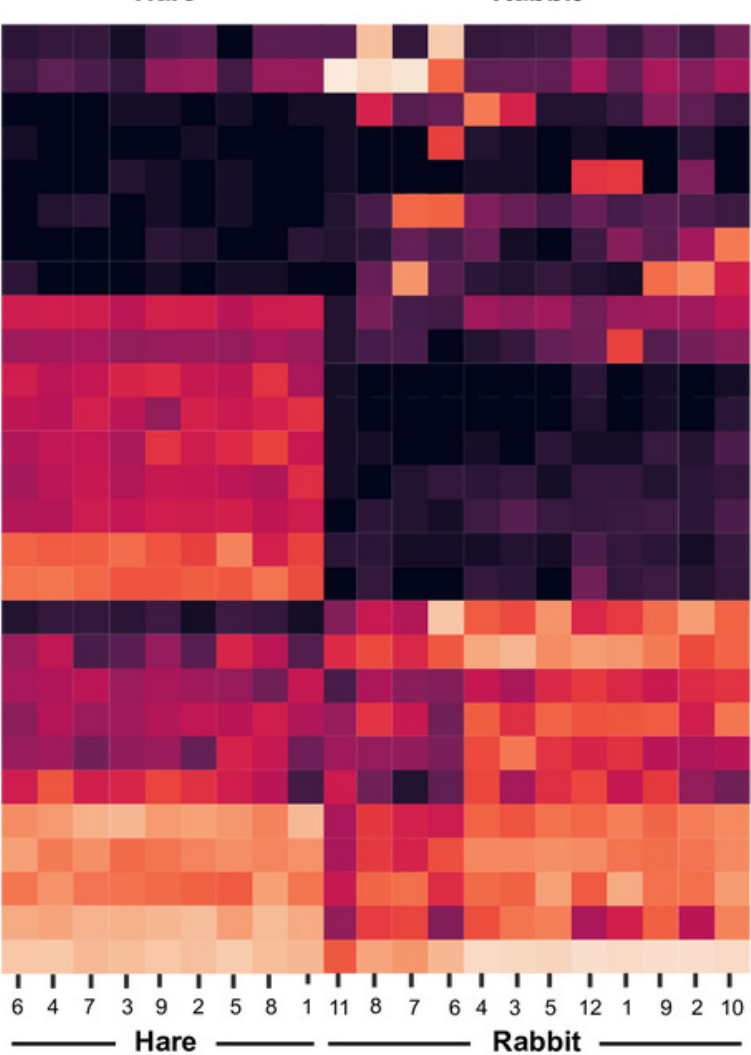




\section{Figure 4}

Faecal microbial diversity of wild hares in their native range of Europe shows Spirochetes to be the third most abundant bacterial phyla.

Raw Illumina sequencing data were obtained from a previous study examining the 16S rRNA diversity in faecal pellets of European brown hares in Europe (24). Reads were processed and classified in parallel with sequencing data from Australia hare faecal samples, using BLAST+ as implemented in QIIME2 against the SILVA_132 reference database. The relative frequency of reads assigned to bacterial phyla present at an abundance greater than $0.5 \%$ were plotted for each sample. Australian hare data presented here are repeated from Figure 2 for ease of comparison. 


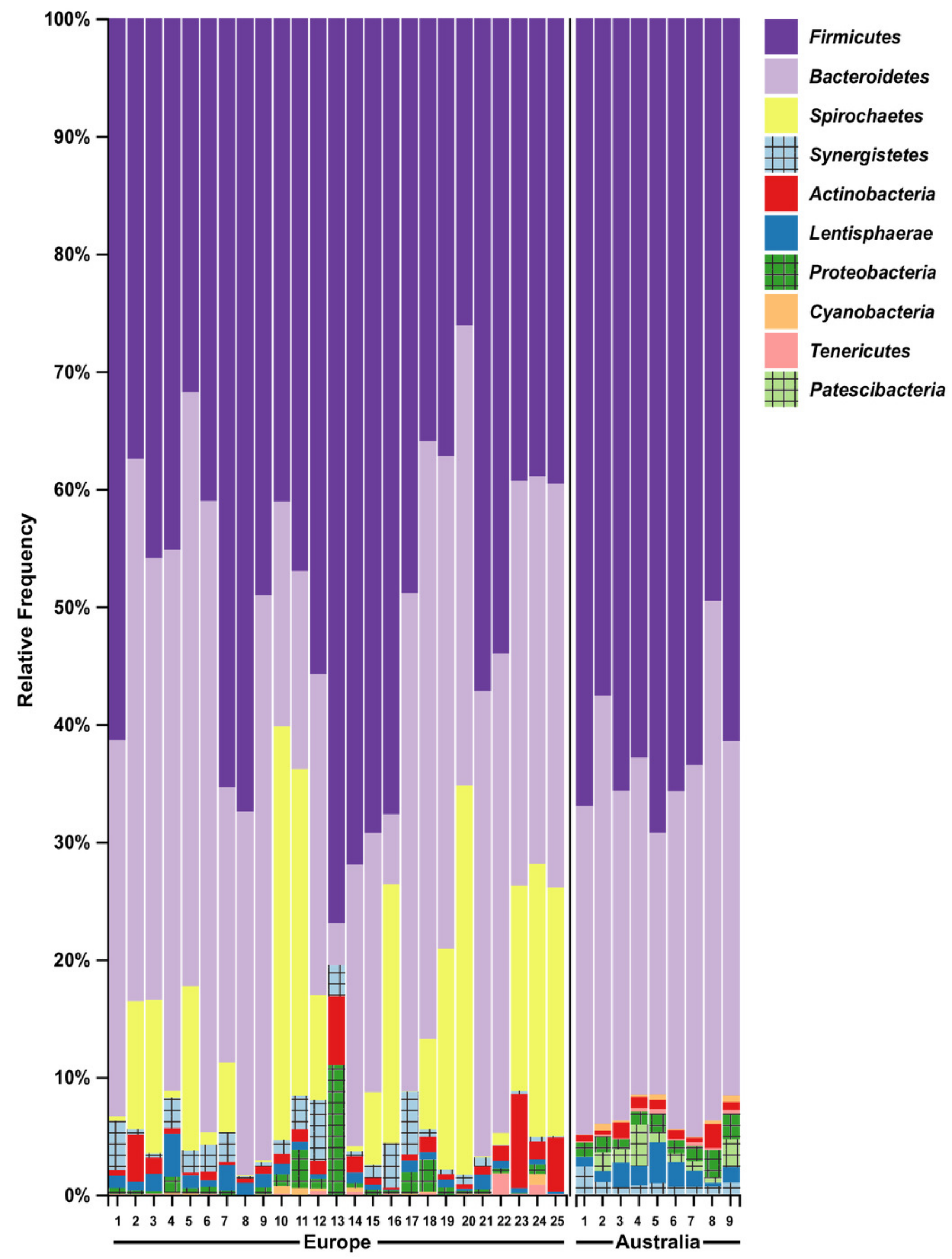

